\title{
Complete Convergence for Arrays of Rowwise Negatively Dependent Random Variables
}

\author{
Fengxiang Feng ${ }^{1,2,}$, Dingcheng Wang ${ }^{1,3, b}$ \\ ${ }^{1}$ School of Mathematical Science, University of Electronic Science and Technology of China, \\ Chengdu, Sichuan 611731, China \\ ${ }^{2}$ College of Science, Guilin University of Technology, Guilin 541004, China \\ ${ }^{3}$ Center of Financial Engineering, Nanjing Audit University, Nanjing 211815, China \\ afengfengxiang2013@163.com, bwangdc@uestc.edu.cn
}

Keywords: Complete convergence, m-ND random variables, Arrays of rowwise m-ND random variables.

\begin{abstract}
In this article, we establish a complete convergence theorem for arrays of rowwise mnegatively dependent (m-ND) random variables. Our results generalize those on complete convergence theorem previously obtained by Hu et al. (1998) and Sung et al. (2005) from independent distributed case to m-ND arrays.
\end{abstract}

\section{Introduction}

The concept of complete convergence of a sequence of random variables was introduced by Hsu and Robbins (1947). Hu et al. (1998) proposed the following general complete convergence of rowwise independent arrays of random variables:

Theorem $\mathbf{A}$ Let $\left\{X_{n i} ; 1 \leq i \leq k_{n}, n \geq 1\right.$ be an array of rowwise independent random variables and $\left\{c_{n}\right\}$ be a sequence of positive real numbers. Suppose that for every $\varepsilon>0$ and some $\delta>0$ :

(i) $\sum_{n=1}^{\infty} c_{n} \sum_{i=1}^{k_{n}} P\left\{\left|X_{n i}\right|>\varepsilon\right\}<\infty$,

(ii) there exists $j \geq 1$ such that

(iii) $\sum_{i=1}^{k_{n}} E X_{n i} I\left\{\left|X_{n i}\right| \leq \delta\right\} \rightarrow 0$ as $n \rightarrow \infty$.

$$
\sum_{n=1}^{\infty} c_{n}\left(\sum_{i=1}^{k_{n}} E X_{n i}^{2} I\left\{\left|X_{n i}\right| \leq \delta\right\}\right)^{j}<\infty,
$$

Then

$$
\sum_{n=1}^{\infty} c_{n} P\left(\left|\sum_{i=1}^{k_{n}} X_{n i}\right|>\varepsilon\right)<\infty \text { forall } \varepsilon>0 .
$$

In this paper we let $\left\{k_{n}, n \geq 1\right\}$ be a sequence of positive integers such that $\lim _{n \rightarrow \infty} k_{n}=\infty$.

The proof of Hu et al. (1998) is mistakenly based on the fact that the assumptions of Theorem A imply convergence in probability of the corresponding partial sums. Hu and Volodin (2000) and $\mathrm{Hu}$ et al. (2003) presented counterexamples to this proof. They mentioned that whether Theorem A is true has remained open. Since then many authors attempted to solve this problem. Hu et al. (2003) and Kuczmaszewskagave (2004) gave partial solution to this question. Sung et al. (2005) completely solved this problem by using a symmetrization procedure and Kruglov et al. (2006) obtained the complete convergence for maximum partial sums by using a submartingale approach.

Recently, Chen et al. (2008) extended Theorem A to the case of arrays of rowwise negatively associated random variables and obtained the complete convergence for maximum partial sums. Hu et al. (2009) obtained complete convergence for maximum partial sums similar to Theorem A for arrays of rowwise m-negatively associated random variables. Qiu et al. (2011) obtained similar result for arrays of rowwise negatively dependent random variables.

Lehmann (1996) introduced the concept of negatively dependent random variables as follows. 
Definition 1. A sequence of random variables $\left\{X_{n} ; n \geq 1\right\}$ is said to be negatively dependent (abbreviated to ND in the following) if for any $n \geq 2$, the following two inequalities hold:

$$
\begin{aligned}
& P\left(X_{1} \leq x_{1}, \cdots, X_{n} \leq x_{n}\right) \leq \prod_{i=1}^{n} P\left(X_{i} \leq x_{i}\right) \\
& P\left(X_{1}>x_{1}, \cdots, X_{n}>x_{n}\right) \leq \prod_{i=1}^{n} P\left(X_{i}>x_{i}\right)
\end{aligned}
$$

for every sequence $\left\{x_{1}, \cdots, x_{n}\right\}$ of real numbers.

Definition 2. Let $m \geq 1$ be a fixed integer. A sequence of random variables $\left\{X_{n} ; n \geq 1\right\}$ is said to be $m$-negatively dependent (abbreviated to $m-N D$ in the following) if for any $n \geq 2$ and $i_{1}, \cdots, i_{n}$ such that $\left|\mathrm{i}_{\mathrm{k}}-\mathrm{i}_{\mathrm{j}}\right| \geq \mathrm{m}$ for all $1 \leq \mathrm{k} \neq \mathrm{j} \leq \mathrm{n}$, we have that $\mathrm{X}_{\mathrm{i}_{1}}, \cdots, \mathrm{X}_{\mathrm{i}_{\mathrm{n}}}$ are negatively dependent.

The concept of $\mathrm{m}-\mathrm{ND}$ random variables is a natural extension from ND random variables (wherein $\mathrm{m}=1)$.

The main purpose of this article is to generalize Theorem A to the case of arrays of rowwise m-ND random variables.

\section{Main results}

Theorem 1. Let $\left\{\mathrm{X}_{\mathrm{ni}} ; 1 \leq \mathrm{i} \leq \mathrm{k}_{\mathrm{n}}, \mathrm{n} \geq 1\right\}$ be an array of rowwise $\mathrm{m}-\mathrm{ND}$ random variables and $\left\{\mathrm{c}_{\mathrm{n}}\right\}$ be a sequence of positive real numbers. Assume that for every $\varepsilon>0$ and some $\delta>0$ :

(i) $\sum_{\mathrm{n}=1}^{\infty} \mathrm{c}_{\mathrm{n}} \sum_{\mathrm{i}=1}^{\mathrm{k}_{\mathrm{n}}} \mathrm{P}\left(\left|\mathrm{X}_{\mathrm{ni}}\right|>\varepsilon\right)<\infty$,

(ii) there exists $\mathrm{j} \geq 1$ such that

Then

$$
\sum_{n=1}^{\infty} c_{n}\left(\sum_{i=1}^{k_{n}} E X_{n i}^{2} I\left\{\left|X_{n i}\right| \leq \delta\right\}\right)^{j}<\infty
$$

$$
\sum_{n=1}^{\infty} c_{n} P\left(\left|\sum_{i=1}^{k_{n}}\left(X_{n i}-E X_{n i} I\left\{\left|X_{n i}\right| \leq \delta\right\}\right)\right|>\varepsilon\right)<\infty \text { forall } \varepsilon>0
$$

\section{Proofs of main results}

In order to prove our results, we need the following lemmas.

Lemma 1. (see Qiu et al. (2011), Lemma 2) Let $\left\{X_{n} ; n \geq 1\right\}$ be a sequence of negatively dependent random variables with $\mathrm{EX}_{\mathrm{n}}=0$ and $\mathrm{EX}_{\mathrm{n}}^{2}<\infty, \mathrm{n} \geq 1$. Let $\mathrm{S}_{\mathrm{n}}=\sum_{\mathrm{i}=1}^{\mathrm{n}} \mathrm{X}_{\mathrm{i}}, \mathrm{B}_{\mathrm{n}}=\sum_{\mathrm{i}=1}^{\mathrm{n}} \mathrm{EX}_{\mathrm{i}}^{2}$. Then for all $\mathrm{x}>0, \mathrm{a}>0$

$$
\begin{gathered}
P\left(S_{n}>x\right) \leq P\left(\max _{1 \leq k \leq n}\left|X_{k}\right|>a\right)+\exp \left\{\frac{x}{a}-\frac{x}{a} \ln \left(1+\frac{x a}{B_{n}}\right)\right\} . \\
P\left(\left|S_{n}\right|>x\right) \leq 2 P\left(\max _{1 \leq k \leq n}\left|X_{k}\right|>a\right)+2 \exp \left\{\frac{x}{a}-\frac{x}{a} \ln \left(1+\frac{x a}{B_{n}}\right)\right\} .
\end{gathered}
$$

Lemma 2. Let $\left\{X_{n} ; n \geq 1\right\}$ be a sequence of $m-N D$ random variables with $\operatorname{EX}_{n}=0$ and $\mathrm{EX}_{\mathrm{n}}^{2}<\infty$, $\mathrm{n} \geq 1$. Let $\mathrm{S}_{\mathrm{n}}=\sum_{\mathrm{i}=1}^{\mathrm{n}} \mathrm{X}_{\mathrm{i}}, \mathrm{B}_{\mathrm{n}}=\sum_{\mathrm{i}=1}^{\mathrm{n}} \mathrm{E} X_{\mathrm{i}}^{2}$. Then for all $\mathrm{n} \geq \mathrm{m}, \mathrm{x}>0$, a $>0$

and

$$
P\left(S_{n}>x\right) \leq m P\left(\max _{1 \leq k \leq n}\left|X_{k}\right|>a\right)+m \exp \left\{\frac{x}{m a}-\frac{x}{m a} \ln \left(1+\frac{x a}{m B_{n}}\right)\right\}
$$

$$
P\left(\left|S_{n}\right|>x\right) \leq 2 m P\left(\max _{1 \leq k \leq n}\left|X_{k}\right|>a\right)+2 m \exp \left\{\frac{x}{m a}-\frac{x}{m a} \ln \left(1+\frac{x a}{m B_{n}}\right)\right\} .
$$

Proof. Given any $1 \leq \mathrm{k} \leq \mathrm{n}$, Let $\mathrm{r}=\left[\frac{\mathrm{n}}{\mathrm{m}}\right]$. Set

$Y_{i}=\left\{\begin{array}{c}X_{i} \quad \text { if } 1 \leq i \leq n \\ 0 \quad \text { if } i>1\end{array}\right.$

and $S_{m k+j}^{\prime}=\sum_{i=0}^{k} Y_{m i+j}$ for $1 \leq j \leq m$. 
Since $\left\{S_{n}>x\right\} \subset\left\{S_{m r+1}^{\prime}>\frac{x}{m}\right\} \cup \cdots \cup\left\{S_{m r+m}^{\prime}>\frac{x}{m}\right\}$,

By Lemma 3.1, we have

$$
\begin{aligned}
& P\left(S_{n}>x\right) \leq \sum_{j=1}^{m} P\left(S_{m r+j}^{\prime}>\frac{x}{m}\right) \\
& \leq \sum_{j=1}^{m} P\left(\max _{0 \leq i \leq r}\left|Y_{m i+j}\right|>a\right)+\sum_{j=1}^{m} \exp \left\{\frac{x}{m a}-\frac{x}{m a} \ln \left(1+\frac{x a}{m \sum_{i=0}^{r} E Y_{m i+j}^{2}}\right)\right\} \\
& \leq m P\left(\max _{1 \leq k \leq n}\left|X_{k}\right|>a\right)+m \exp \left\{\frac{x}{m a}-\frac{x}{m a} \ln \left(1+\frac{x a}{m B_{n}}\right)\right\} .
\end{aligned}
$$

If we consider $-X_{n}$ instead of $X_{n}$ in the arguments above, by a similar way we get

Therefore,

$$
P\left(-S_{n}>x\right) \leq m P\left(\max _{1 \leq k \leq n}\left|X_{k}\right|>a\right)+m \exp \left\{\frac{x}{m a}-\frac{x}{m a} \ln \left(1+\frac{x a}{m B_{n}}\right)\right\} \text {. }
$$

$$
P\left(\left|S_{n}\right|>x\right) \leq 2 m P\left(\max _{1 \leq k \leq n}\left|X_{k}\right|>a\right)+2 m \exp \left\{\frac{x}{m a}-\frac{x}{m a} \ln \left(1+\frac{x a}{m B_{n}}\right)\right\} .
$$

Lemma 3. Let $\mathrm{Y}_{\mathrm{ni}}=\delta \mathrm{I}\left\{\mathrm{X}_{\mathrm{ni}}>\delta\right\}+\mathrm{X}_{\mathrm{ni}} \mathrm{I}\left\{\left|\mathrm{X}_{\mathrm{ni}}\right| \leq \delta\right\}-\delta \mathrm{I}\left\{\mathrm{X}_{\mathrm{ni}}<-\delta\right\}$. Under the conditions of Theorem 2.1, we have

$$
\max _{1 \leq i \leq k_{n}}\left|E Y_{n i}\right| \leq\left(\sum_{i=1}^{k_{n}} E X_{n i}^{2} I\left\{\left|X_{n i}\right| \leq \delta\right\}\right)^{\frac{1}{2}}+\delta \sum_{i=1}^{k_{n}} P\left(\left|X_{n i}\right|>\delta\right) .
$$

Proof. $\left\{\mathrm{Y}_{\mathrm{ni}}, 1 \leq \mathrm{i} \leq \mathrm{k}_{\mathrm{n}}, \mathrm{n} \geq 1\right\}$ is an array of rowwise $\mathrm{m}-\mathrm{ND}$ random variables.

$$
\begin{aligned}
\max _{1 \leq \mathrm{i} \leq \mathrm{k}_{\mathrm{n}}}\left|\mathrm{EY}_{\mathrm{ni}}\right| & \leq \max _{1 \leq \mathrm{i} \leq \mathrm{k}_{\mathrm{n}}} \mathrm{E}\left|\mathrm{Y}_{\mathrm{ni}}\right|=\max _{1 \leq \mathrm{i} \leq \mathrm{k}_{\mathrm{n}}} \mathrm{E}\left|\mathrm{X}_{\mathrm{ni}} \mathrm{I}\left\{\left|\mathrm{X}_{\mathrm{ni}}\right| \leq \delta\right\}+\delta \mathrm{I}\left\{\mathrm{X}_{\mathrm{ni}}>\delta\right\}-\delta \mathrm{I}\left\{\mathrm{X}_{\mathrm{ni}}<-\delta\right\}\right| \\
& \leq \max _{1 \leq \mathrm{i} \leq \mathrm{k}_{\mathrm{n}}}\left(\mathrm{E}\left|\mathrm{X}_{\mathrm{ni}} \mathrm{I}\left\{\left|\mathrm{X}_{\mathrm{ni}}\right| \leq \delta\right\}\right|+\delta \mathrm{P}\left(\left|\mathrm{X}_{\mathrm{ni}}\right|>\delta\right)\right) \\
& \leq \max _{1 \leq \mathrm{i} \leq \mathrm{k}_{\mathrm{n}}}\left(\mathrm{EX}_{\mathrm{ni}}^{2} \mathrm{I}\left\{\left|\mathrm{X}_{\mathrm{ni}}\right| \leq \delta\right\}\right)^{\frac{1}{2}}+\delta \sum_{\mathrm{i}=1}^{\mathrm{k}_{\mathrm{n}}} \mathrm{P}\left(\left|\mathrm{X}_{\mathrm{ni}}\right|>\delta\right) \\
& \leq\left(\sum_{\mathrm{i}=1}^{\frac{1}{2}} \mathrm{EX}_{\mathrm{ni}}^{2} \mathrm{I}\left\{\left|\mathrm{X}_{\mathrm{ni}}\right| \leq \delta\right\}\right)^{\mathrm{k}_{\mathrm{n}}}+\delta \sum_{\mathrm{i}=1}^{\mathrm{P}} \mathrm{P}\left(\left|\mathrm{X}_{\mathrm{ni}}\right|>\delta\right) .
\end{aligned}
$$

Proof. Proof of Theorem 2.1 Let

$$
\begin{gathered}
\mathrm{Y}_{\mathrm{ni}}=\delta \mathrm{I}\left\{\mathrm{X}_{\mathrm{ni}}>\delta\right\}+\mathrm{X}_{\mathrm{ni}} \mathrm{I}\left\{\left|\mathrm{X}_{\mathrm{ni}}\right| \leq \delta\right\}-\delta \mathrm{I}\left\{\mathrm{X}_{\mathrm{ni}}<-\delta\right\} \quad \text { and } \\
\mathrm{Y}_{\mathrm{ni}}^{\prime}=\delta \mathrm{I}\left\{\mathrm{X}_{\mathrm{ni}}>\delta\right\}-\delta \mathrm{I}\left\{\mathrm{X}_{\mathrm{ni}}<-\delta\right\} \quad \text { and } 1 \leq \mathrm{i} \leq \mathrm{k}_{\mathrm{n}}, \mathrm{n} \geq 1 .
\end{gathered}
$$

$\left\{\mathrm{Y}_{\mathrm{ni}}, 1 \leq \mathrm{i} \leq \mathrm{k}_{\mathrm{n}}, \mathrm{n} \geq 1\right\}$ is an array of rowwise $\mathrm{m}-\mathrm{ND}$ random variables. Note that

$$
\begin{aligned}
& \mathrm{P}\left(\left|\sum_{i=1}^{\mathrm{k}_{\mathrm{n}}}\left(\mathrm{X}_{\mathrm{ni}}-\mathrm{EX}_{\mathrm{ni}} \mathrm{I}\left\{\left|\mathrm{X}_{\mathrm{ni}}\right| \leq \delta\right\}\right)\right|>\varepsilon\right) \\
& \leq \sum_{\mathrm{i}=1}^{\mathrm{k}_{\mathrm{n}}} \mathrm{P}\left(\left|\mathrm{X}_{\mathrm{ni}}\right|>\delta\right)+\mathrm{P}\left(\left|\sum_{\mathrm{i}=1}^{\mathrm{k}_{\mathrm{n}}}\left(\mathrm{X}_{\mathrm{ni}} \mathrm{I}\left\{\left|\mathrm{X}_{\mathrm{ni}}\right| \leq \delta\right\}-\mathrm{EX}_{\mathrm{ni}} \mathrm{I}\left\{\left|\mathrm{X}_{\mathrm{ni}}\right| \leq \delta\right\}\right)\right|>\varepsilon\right) \\
& =\sum_{\mathrm{i}=1}^{\mathrm{k}_{\mathrm{n}}} \mathrm{P}\left(\left|\mathrm{X}_{\mathrm{ni}}\right|>\delta\right)+\mathrm{P}\left(\left|\sum_{\mathrm{i}=1}^{\mathrm{k}_{\mathrm{n}}}\left(\mathrm{Y}_{\mathrm{ni}}-\mathrm{EY}_{\mathrm{ni}}-\mathrm{Y}_{\mathrm{ni}}^{\prime}+\mathrm{EY}_{\mathrm{ni}}^{\prime}\right)\right|>\varepsilon\right) \\
& \leq \sum_{\mathrm{i}=1}^{\mathrm{k}_{\mathrm{n}}} \mathrm{P}\left(\left|\mathrm{X}_{\mathrm{ni}}\right|>\delta\right)+\mathrm{P}\left(\left|\sum_{\mathrm{i}=1}^{\mathrm{k}_{\mathrm{n}}}\left(\mathrm{Y}_{\mathrm{ni}}^{\prime}-\mathrm{EY}_{\mathrm{ni}}^{\prime}\right)\right|>\varepsilon / 2\right)+\mathrm{P}\left(\left|\sum_{\mathrm{i}=1}^{\mathrm{k}_{\mathrm{n}}}\left(\mathrm{Y}_{\mathrm{ni}}-\mathrm{EY} \mathrm{Y}_{\mathrm{ni}}\right)\right|>\varepsilon / 2\right) \\
& \leq \sum_{\mathrm{i}=1}^{\mathrm{k}_{\mathrm{n}}} \mathrm{P}\left(\left|\mathrm{X}_{\mathrm{ni}}\right|>\delta\right)+\mathrm{C} \sum_{\mathrm{i}=1}^{\mathrm{k}_{\mathrm{n}}} \mathrm{P}\left(\left|\mathrm{X}_{\mathrm{ni}}\right|>\delta\right)+\mathrm{P}\left(\left|\sum_{\mathrm{i}=1}^{\mathrm{k}_{\mathrm{n}}}\left(\mathrm{Y}_{\mathrm{ni}}-\mathrm{EY}_{\mathrm{ni}}\right)\right|>\varepsilon / 2\right)
\end{aligned}
$$


Hence, by conditions (i), it is sufficient to prove that

$$
\sum_{n=1}^{\infty} c_{n} P\left(\left|\sum_{i=1}^{k_{n}}\left(Y_{n i}-E Y_{n i}\right)\right|>\varepsilon / 2\right)<\infty .
$$

\section{Acknowledgments}

This work was financially supported by National Natural Science Foundation of China (71271042).

\section{References}

[1] P. Hsu, H. Robbins, Complete convergence and the law of large numbers, Proc. Natl. Acad. Sci. USA. 33 (1947) 25-31.

[2].T.-C. Hu, D. Szynal, A. Volodin, A note on complete convergence for arrays, Statist. Probab. Lett. 38 (1998) 27-31.

[3].T.-C. Hu, A. Volodin, Addendum to "A note on complete convergence for arrays", Statist. Probab. Lett. 47(2000) 209-211.

[4].T.-C. Hu, M. Ord[Trial mode]ez Cabrera, S.H. Sung, A. Volodin, Complete convergence for arrays of rowwise independent random variables, Commun. Korean Math. Soc. 18 (2003) 375383.

[5].A. Kuczmaszewska, on some conditions for complete convergence for arrays, Statist. Probab. Lett. 66 (2004) 399-405.

[6].S.H. Sung, T.-C. Hu, A.I. Volodin, More on complete convergence for arrays, Statist. Probab. Lett. 71 (2005) 303-311.

[7].V.M. Kruglov, A.I. Volodin, T.-C. Hu, on complete convergence for arrays, Statist. Probab. Lett. 76 (2006) 1631-1640.

[8].P. Chen, T.-C. Hu, X. Liu, A. Volodin, on complete convergence for arrays of row-wise negatively associated random variables, Theor. Probab. Appl. 52 (2) (2008) 323-328.

[9].T.-C. Hu, C.-Y Chiang, R.L. Taylor, on complete convergence for arrays of rowwise m-negatively associated random variables, Nonlinear. Anal. 71(2009) 1075-1081.

[10].D. Qiu, K.-C. Chang, R.G. Antonini, A. Volodin, on the strong rates of convergence for arrays of rowwise negatively dependent random variables, Stochastic Anal. Appl. 29 (2011) 375-385.

[11].E. Lehmann, Some concepts of dependence. Ann. Math. Statist. 37 (1996) 1137-1153.

[12].Y. Wu, M.O. Cabrera, A. Volodin, on limiting behavior for arrays of rowwise negatively orthant dependent random variables. J. Korean. Stat. Soc. 42(2013) 61-70. 